\title{
The Effect of Lipopolysaccharide Composition on the Ultrastructure of Pseudomonas aeruginosa
}

\author{
By PAULINE M. MEADOW AND PAUL L. WELLS \\ Department of Biochemistry, Univcrsity College London, London WC1E $6 B T$ \\ MIRJA SALKINOJA-SALONEN AND EEVA-LIISA NURMIAHO \\ Department of General Microbiology, University of Helsinki, SF-00100, \\ Helsinki 10, Finland
}

(Received 30 September 1977)

\begin{abstract}
The surface structure of Pseudomonas aeruginosa PACl and PACl R and of lipopolysaccharidedefective mutants derived from them was studied by negative-staining and thin-section electron microscopy and compared with that of a rough mutant with wild-type lipopolysaccharide. The rough mutant and the parent strains had fairly smooth outer layers. Negatively stained preparations of all the mutants lacking polymerized O-antigenic sidechains, including a semi-rough mutant, showed numerous blebs on the surface. In thin sections of these mutants occasional extrusions from the surface were seen. They appeared to consist of material extruded from the outer membrane, but there was no evidence to suggest they were complete unit membranes. Polymerized O-antigenic side-chains in the lipopolysaccharide appear to be required to produce the wild-type appearance of the outer membrane in $P$. aeruginosa.
\end{abstract}

\section{INTRODUCTION}

In the Enterobacteriaceae, phenotypically rough mutants have defective lipopolysaccharides (LPSs) lacking the O-antigenic side-chains and often parts of the core (for review, see Lüderitz et al., 1971). Such mutants of Salmonella typhimurium have an unstable outer membrane, and extruded blebs of incomplete LPS can be seen by electron microscopy (Smit, Kamio \& Nikaido, 1975; Irvin et al., 1975). In Pseudomonas aeruginosa, LPS-defective mutants do not produce phenotypically rough colonies and mutants giving rough colonies on plates appear to have wild-type LPS (Koval \& Meadow, 1977). It was thus interesting to see whether the ultrastructure of the surface of LPS-defective mutants of $P$. aeruginosa was different from that of the parent strain and other mutants with wild-type LPS.

\section{METHODS}

Organisms and growth conditions. Pseudomonas aeruginosa PACl (NCIB 10848) which belongs to Habs serotype 3, PAClR which is a spontaneous mutant isolated from it as resistant to phage PS1, the LPSdefective mutants PAC605 and PAC608, and the phenotypically rough mutant PAC307 have all been described before (Koval \& Meadow, 1977). Pseudomonas aeruginosa PAc610 is a spontaneous mutant isolated from PAC605 by T. L. Pitt (Cross Infection Reference Laboratory, Colindale, London) by selecting for resistance to dilutions of human sera. All cultures were grown at $37^{\circ} \mathrm{C}$ either with shaking in flasks containing onefifth their volume of nutrient broth (Oxoid no. 2) or on plates of nutrient agar $(1.5 \%, w / v)$.

Isolation and analysis of LPS. LPS was isolated from bacterial walls, partially hydrolysed, separated on columns of Sephadex G75, and the glucose, rhamnose, heptose, phosphate and amino compounds present in each fraction were determined, all as described previously (Koval \& Meadow, 1977). 


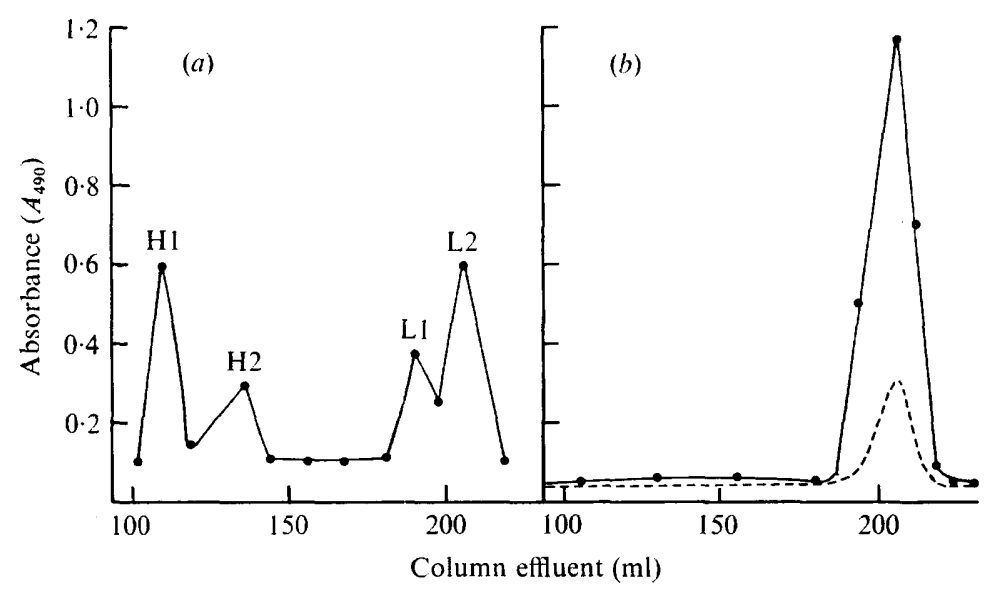

Fig. 1. Fractionation of polysaccharide obtained from partially degraded LPS from $P$. aeruginosa: (a) PAC1R, (b) PAC610 (-) and PAC605 (-- ). LPS $(30 \mathrm{mg})$ was hydrolysed with $1 \%(\mathrm{v} / \mathrm{v})$ acetic acid for $1 \mathrm{~h}$ and the hydrolysate was eluted from a column $(2.5 \times 70 \mathrm{~cm})$ of Sephadex G75 with pyridine/acetic acid buffer, $\mathrm{pH} 5 \cdot 4$. Fractions $(4 \mathrm{ml})$ were analysed for total carbohydrate by the phenol/sulphuric acid method.

Electron microscopy. (i) Negative staining. A drop of the sample was applied to a copper grid coated with Formvar and carbon and the excess was removed. The stain [phosphotungstic acid $(1 \%, \mathrm{w} / \mathrm{v})$ adjusted to pH 6.5 with $\mathrm{KOH}$ ] was applied immediately and the excess was again removed with filter paper.

(ii) Thin sections. Bacteria in the exponential growth phase were harvested by centrifugation $(10000 \mathrm{~g})$. The pellets were prefixed in $0 \cdot 1$ M-sodium phosphate buffer $(\mathrm{pH} 7 \cdot 2)$ containing glutaraldehyde $(3 \%$, w/v) and then washed three times in the buffer. Postfixation was for $2 \mathrm{~h}$ at room temperature in the same buffer containing osmium tetroxide $(1 \%, \mathrm{w} / \mathrm{v})$. The specimens were dehydrated through a series of concentrations of ethanol and propylene oxide and then embedded in Epon 812. Thin sections were cut with a diamond knife on a Sorvall Porter-Blum MT-2 ultramicrotome and double-stained with uranyl acetate and lead citrate. The grids were examined in a Jeol JEM 100B electron microscope at an operating voltage of $80 \mathrm{kV}$.

\section{RESULTS AND DISCUSSION}

\section{Characterization of the mutants}

The serum-resistant mutant PAC610 appeared to have a defective LPS since it did not react with Habs type 3 antiserum. Separation of its partially degraded polysaccharide on Sephadex G75 yielded a single peak as detected by the phenol/sulphuric acid reagent. The polysaccharide appeared to be similar to that from PAC605 from which PAc610 had been derived except that there was more carbohydrate (Fig. 1b). As expected from the lack of $\mathrm{O}$-antigenicity, the degraded polysaccharide contained neither the high molecular weight peaks derived from the $\mathrm{O}$-antigenic side-chains $(\mathrm{H} 1$ and $\mathrm{H} 2$ ) nor the leading shoulder (L1) on the low molecular weight fraction, all of which are produced from the wild-type LPS (Fig. 1a). Analysis of the L2 peak obtained from PAC610 showed that it contained (molar ratios in parentheses) glucose $(4 \cdot 5)$, rhamnose $(1 \cdot 8)$, heptose $(2 \cdot 4)$, galactosamine $(1 \cdot 0)$ and alanine $(1 \cdot 3)$. It thus appeared to contain the core components found in the wild-type LPS (Koval \& Meadow, 1977) in approximately the same molar ratios except for glucose (wildtype $5 \cdot 5)$.

The LPS composition and some other properties of the mutants studied are summarized in Table 1 .

\section{Electron microscopy}

Negative staining. The LPS-defective mutants were clearly distinguishable from the wild type by negative-staining electron microscopy (Fig. 2). The surface of the parent strain appeared fairly smooth. Many preparations showed extraneous globular material of 
Table 1. Properties of strains used

\begin{tabular}{|c|c|c|c|c|c|c|}
\hline Strain & PAC1 & PAClR & PAC307 & PAC608 & PAC610 & PAC 605 \\
\hline Parent strain & & PACl & PACl & PAClR & PAC605 & PAClR \\
\hline Phenotype selected & & $\begin{array}{c}\text { Resistance } \\
\text { to PS1 }\end{array}$ & Amidase $^{-}$ & $\begin{array}{c}\text { Resistance to } \\
\text { pyocin P16 }\end{array}$ & $\begin{array}{l}\text { Serum- } \\
\text { resistant }\end{array}$ & $\begin{array}{c}\text { Resistance to } \\
\text { pyocin P16 }\end{array}$ \\
\hline Colony type & Smooth & Smooth & Rough & Smooth & Smooth & Smooth \\
\hline Habs serotype & 3 & 3 & 3 & 3 & NT & NT \\
\hline \multicolumn{7}{|c|}{ LPS fractions present* } \\
\hline $\mathrm{H} 1, \mathrm{H} 2$ & + & + & + & 0 & 0 & 0 \\
\hline L1 & + & + & + & + & 0 & 0 \\
\hline $\mathrm{L} 2$ & + & + & + & + & + & + \\
\hline
\end{tabular}

NT, Not typable; + , present; 0 , absent.

* The fractions are those shown in Fig. 1.

approximately $20 \mathrm{~nm}$ diameter attached to the surface of the bacteria, the flagella and the pili. This material may have been fragments of the outer membrane which were extruded from the bacterial surface and which, because of their lipophilicity, attached to the hydrophobic surfaces of the organism. Such material has been seen in several different species, and in Escherichia coli has been identified chemically as fragments of outer membrane (Hoekstra et al., 1976). The large amounts present in some of our preparations may reflect the relatively fragile walls of $P$. aeruginosa, which in turn may depend in part on the small amounts of lipoprotein linking the outer membrane to the peptidoglycan as compared with the amounts found in E. coli (for a discussion of wall structure in Pseudomonas, see Meadow, 1975).

The parent strain PACl, PAC1R and PAC307 as well as other mutants (not listed) known to have complete wild-type LPS, all appeared similar by negative staining (see Fig. $2 a$ ). In contrast all mutants lacking polymerized $\mathrm{O}$-antigenic side-chains showed typical extrusions from the wall (see Fig. $2 b$ ). The extrusions resembled those found in rough strains of S. typhimurium LT2 (Irvin et al., 1975; Smit et al., 1975). Similar blebs can be induced by polymyxin treatment in $P$. aeruginosa P29 (Koike, Iida \& Matsuo, 1969) and in smooth strains of $S$. typhimurium (Lounatmaa, Mäkelä \& Sarvas, 1976). In $P$. aeruginosa PACl and mutants derived from it, the blebs were always present in mutants lacking the polymerized O-antigenic side-chains of the LPS. We could detect no differences either in appearance or in the number of blebs in mutants with additional core defects. The mutant shown in Fig. 2(b), PAC610, apparently contained an almost complete core, yet its appearance was indistinguishable both from the most defective mutant PAC605 and the least defective PAC608. The latter contains not only a complete LPS core, but more of the L1 fraction (core polysaccharide with a single unit of antigenic side-chain) than the wild type (Koval \& Meadow, 1977). It is thus similar to the semi-rough strains found in Salmonella (Naide et al., 1965). Although it had enough O-antigenic material to agglutinate with Habs type 3 antiserum (Koval \& Meadow, 1977), there was apparently not enough polymerization to prevent the formation of the characteristic surface blebs of the LPS-defective mutants.

Five mutants of PACl whose LPSs contain polymerized O-antigenic side-chains and six LPS-defective mutants have now been examined. All those yielding $\mathrm{H} 1$ fractions after partial hydrolysis of the LPS looked like PAC1 (Fig. $2 a$ ) whereas all those lacking this fraction looked like PAC610 (Fig. $2 b$ ). PAC307, the mutant with rough colonial morphology but wild-type LPS, had a smooth outer layer like PAC1, but the cells were often longer and the rough phenotype may have been caused by some defect in cell division.

Thin sections. Thin sections of PACl and PAClR (Fig. $3 a$ ) showed the typical unit membrane appearance of Gram-negative bacteria with no additional extrusions. The phenotypically rough mutant PAC307 appeared similar except that there was some evidence for 

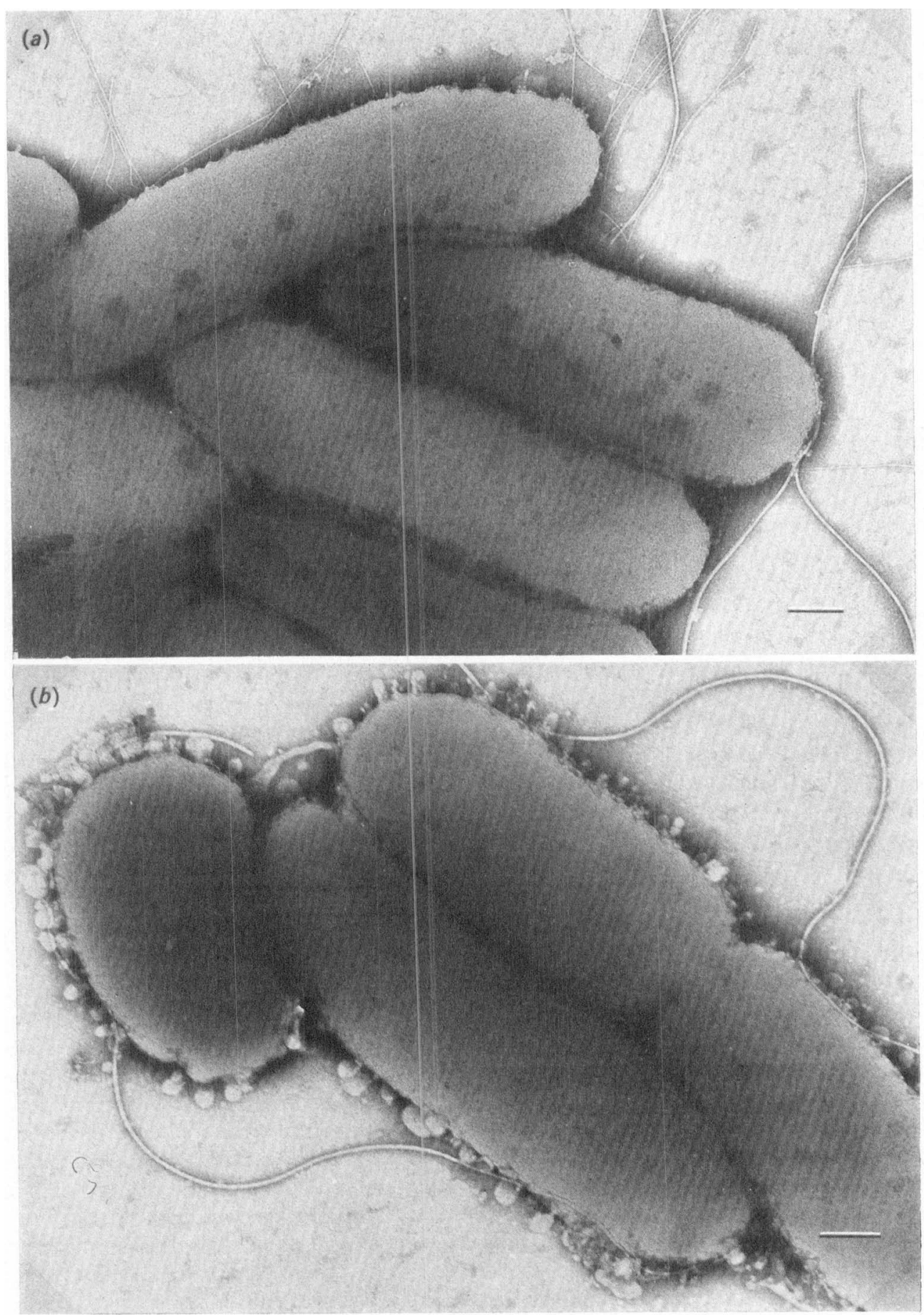

Fig. 2. Surface structure of $P$. aeruginosa: (a) PAC1, (b) PAC610. The preparations were stained with phosphotungstate. Bar markers represent $200 \mathrm{~nm}$. 

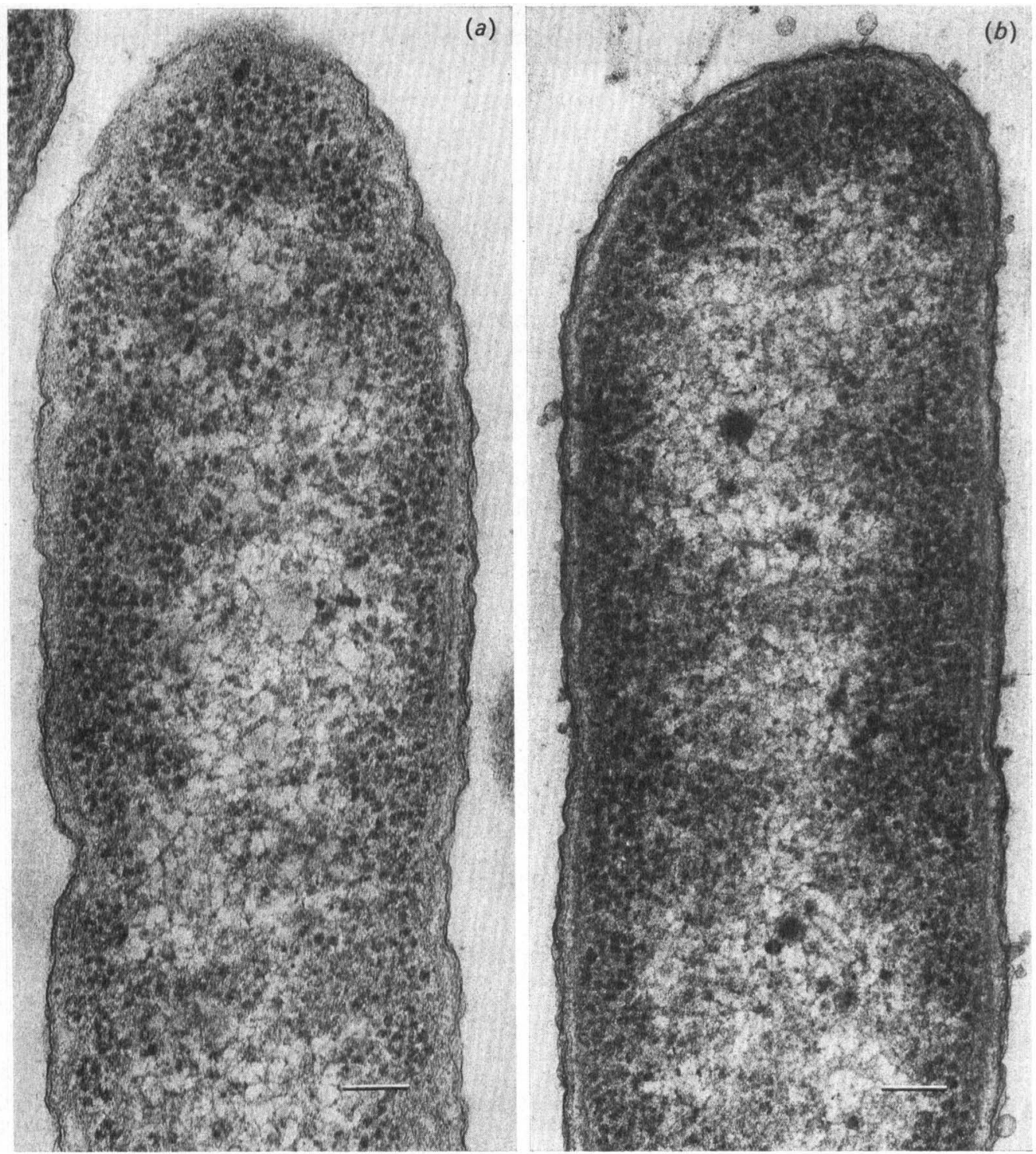

Fig. 3. Thin sections of $P$. aeruginosa: $(a)$ PAC1R, $(b)$ PAC605. The bacteria were prefixed with glutaraldehyde and postfixed with osmium tetroxide. The sections were double-stained with uranyl acetate and lead citrate. Bar markers represent $100 \mathrm{~nm}$.

a delay in cell separation, supporting the tentative conclusion that this mutant is defective in cell division. In thin sections of each of the LPS-defective mutants there were a few small vesicles varying in size from about 20 to $40 \mathrm{~nm}$. They appeared to extrude from the outer membrane (Fig. $3 b$ ). They were fewer in number and considerably smaller than the blebs seen in negatively stained preparations, but their existence was limited to those mutants in which blebs had been seen. The small number of vesicles visible in thin sections may result from their small size so that the bleb seldom fitted into the plane of the section. In addition the washing procedures involved in preparing samples for thin sections might be expected to lead to loss of loosely associated material and to extraction of lipid therefrom. The latter together with the poor staining of polysaccharide material by the procedure used would explain their small size. We could see no sign of unit membrane structure in the vesicles 
although this was visible in a few sections where the outer membrane had detached from the bacteria completely and had curled into the characteristic cylinders found in other species (Nakamura \& Mizushima, 1975; Costerton, Ingram \& Cheng, 1974).

We conclude therefore that in $P$. aeruginosa a rough colonial morphology does not necessarily reflect changes in outer membrane surface structure, but that defects in LPS synthesis preventing synthesis or attachment of polymeric O-antigenic side-chains result in a rough outer membrane surface and the formation of lipophilic blebs. Undulation is sometimes seen on the surface of wild-type S. typhimurium and P. aeruginosa PAo if acrolein is present in the fixative (Gilleland \& Murray, 1976; Lounatmaa, 1977). Even after glutaraldehyde fixation, wild-type Salmonella sometimes show undulation of the surface after growth on solid media rich in salts (K. Lounatmaa, personal communication). Strains of $P$. aeruginosa $\mathrm{PAC}$ with wild-type LPS had the type of appearance shown in Fig. 2(a) whether grown on solid or in liquid media.

It is perhaps surprising that despite the gross alterations in surface structure seen in the LPS-defective mutants of $P$. aeruginosa PAC1 described here, none seemed to be affected in their general stability, growth rate or metabolism and only the most defective mutant PAC605 showed increased sensitivity to any antibiotics (Koval \& Meadow, unpublished).

We thank Dr Kari Lounatmaa for help in the interpretation of the electron microphotographs and Mr T. L. Pitt for mutant PAc610. M. S-S. and E.-L. N. thank the Suomalainen Tiedeakatemia (Jenny ja Antti Wihurin Rahasto) for financial support and Miss Ulla Piililaa for technical assistance.

\section{REFERENCES}

Costerton, J. W., Ingram, J. M. \& Cheng, K.-J. (1974). Structure and function of the cell envelope of Gram-negative bacteria. Bacteriological Reviews 38, 87-110.

Gilleland, H. E. Jr \& Murray, R. G. E. (1976). Ultrastructural study of polymyxin resistant isolates of Pseudomonas aeruginosa. Journal of Bacteriology 125, 267-281.

Hoekstra, D., van der LaAn, J. W., de LeiJ, L. \& Witholt, B. (1976). Release of outer membrane fragments from normally growing Escherichia coli. Biochimica et biophysica acta 455, 889--899.

Irvin, R. T., Chatterjee, A. K., Sanderson, K. E. \& Costerton, J. W. (1975). Comparison of the cell envelope structure of a lipopolysaccharidedefective (heptose-deficient) strain and a smooth strain of Salmonella typhimurium. Journal of Bacteriology 124, 930-941.

KoiKe, M., IidA, K. \& Matsuo, T. (1969). Electron microscopic studies on the mode of action of polymyxin. Journal of Bacteriology 97, 448-452.

Koval, S. F. \& Meadow, P. M. (1977). The isolation and characterization of lipopolysaccharidedefective mutants of Pseudomonas aeruginosa PAC1. Journal of General Microbiology 98, 387398.

LOUNATMAA, K. (1977). Effect of acrolein-glutaraldehyde on the outer membrane of Salmonella typhimurium smooth and rough strains. FEMS Microbiology Letters (in the Press).
Lounatmaa, K., Mäkelä, P. H. \& Sarvas, M (1976). Effect of polymyxin on the ultrastructure of the outer membrane of wild type and polymyxinresistant strains of Salmonella. Journal of Bacteriology 127, 1400-1407.

Lüderitz, O., WestPhal, O., Staub, A. M. \& NikAIDo, H. (1971). Isolation and chemical and immunological characterisation of bacterial lipopolysaccharides. In Microbial Toxins, vol. IV, pp. 145-233. Edited by G. Weinbaum, S. Kadis \& S. J. Ajl. London and New York: Academic Press.

Meadow, P. M. (1975). Wall and membrane structure in the genus Pseudomonas. In Genetics and Biochemistry of Pseudomonas, pp. 67-98. Edited by P. H. Clarke \& M. H. Richmond. London: John Wiley.

NaIde, Y., Nikaido, H., Mäkelä, P. H., WILkINSON, R. G. \& STOCKer, B. A. D. (1965). Semirough strains of Salmonella. Proceedings of the National Academy of Sciences of the United States of America 53, 147-153.

Nakamura, K. \& Mizushima, S. (1975). In vitro assembly of the membranous vesicles from $E$. coli outer membrane components. Biochimica et biophysica acta 413, 371-393.

Smit, J., Kamio, Y. \& Nikaido, H. (1975). Outer membrane of Salmonella typhimurium: chemical analysis and freeze-fracture studies with lipopolysaccharide mutants. Journal of Bacteriology 124, 942-958. 